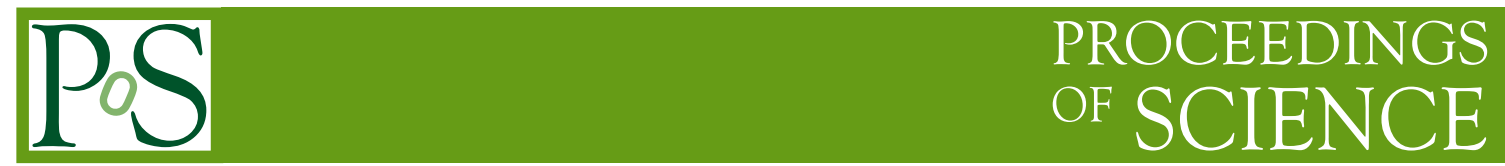

\title{
Upgrade of ALICE forward detectors
}

\section{Maciej Slupecki* ${ }^{\dagger}$}

Department of Physics, University of Jyvaskyla, P.O. Box 35, FI-40014 University of Jyvaskyla, Finland

E-mail: maciej.slupecki@cern.ch

\begin{abstract}
ALICE will upgrade its subsystems in 2019-2020 to fully benefit from the increased collision rate and luminosity of the LHC at CERN. Two new forward detectors will be installed at the core of ALICE: the Fast Interaction Trigger (FIT) and the Muon Forward Tracker (MFT). Additionally, during the shutdown between Run 3 and Run 4, there will be an opportunity to install the Forward Calorimeter (FoCal). This paper describes these new forward detectors, their physics justification and goals, selected design features along with performance of detector prototypes and simulated performance figures.
\end{abstract}

The 39th International Conference on High Energy Physics (ICHEP2018)

4-11 July, 2018

Seoul, Korea

\footnotetext{
* Speaker.

${ }^{\dagger}$ on behalf of the ALICE Collaboration
} 


\section{Introduction}

The LHC is entering a two-year upgrade period of Long Shutdown 2 (LS2) in December 2018. After that the $\mathrm{Pb}-\mathrm{Pb}$ instantaneous luminosity in Run 3 will increase by a factor of $5-6$. The upgraded ALICE experiment [1] aims to record the minimum-bias $\mathrm{Pb}-\mathrm{Pb}$ interactions at the target rate of $50 \mathrm{kHz}$, that is 50 times more than possible with the present setup. To cope with such a dramatic change in the running conditions and to achieve its physics objectives, ALICE will implement a faster readout and record all of the minimum-bias events. Moreover, tracking precision at mid- and forward rapidity will be improved, while preserving unique particle identification capabilities down to very low $p_{\mathrm{T}}$. In addition to the new ITS tracker and improvements of the TPC, two new forward detectors will be installed during LS2: the Fast Interaction Trigger (FIT) and the Muon Forward Tracker (MFT). Figure 1 shows CAD drawings of these detectors.
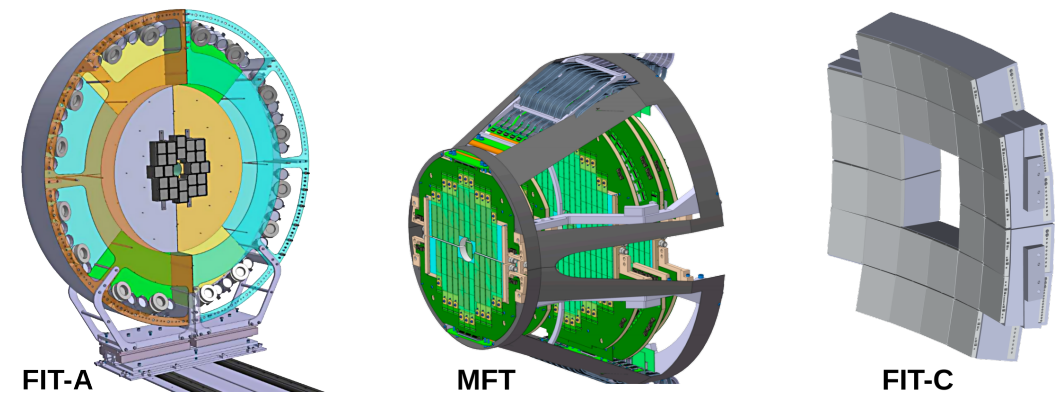

Figure 1: Simplified engineering drawings of the MFT (middle) and the two arms of the FIT detector.

\section{Fast Interaction Trigger (FIT)}

The FIT is composed of two detectors centered around the beam pipe on both sides of the interaction point (IP). FIT-A, shown in Fig. 1 (left), is located $3.3 \mathrm{~m}$ from the IP and covers the pseudorapidity interval of $2.2<\eta<5$.0. It consists of a large segmented plastic scintillator and an array of Cherenkov modules. The Cherenkov array of FIT-C, shown in Fig. 1 (right), is curved to maintain constant distance $(0.82 \mathrm{~m})$ from the modules to the IP. It covers $-3.4<\eta<-2.3$. For historical reasons, the scintillator ring is referred to as V0+ and the Cherenkov array, as $\mathrm{T} 0+$.

A single T0+ Cherenkov module consists of four optically separated quartz radiators $(26.5 \times$ $26.5 \times 20 \mathrm{~mm}^{3}$ each) coupled to a modified Planacon XP85012 MCP-PMT [2]. The selected model was found to be the best in terms of price, performance, compact size, and stability of operation. The cross-talk between the channels has been reduced by modifications of the PCB backplane. The segmented anode of a modified Planacon is grouped into quadrants matching the coverage of the individual radiators and converting each module into four independent detector channels (pixels). The intrinsic time resolution of each pixel is better than 13 ps. The full-chain single-MIP resolution is below 50 ps. Each detector channel covers the dynamic range of 1 to 500 MIPs.

The V0+ is a $1.48 \mathrm{~m}$ diameter scintillator disk, divided symmetrically into $45^{\circ}$ octants. The octants are subdivided into 5 rings of increasing radii so that each ring has a similar pseudorapidity coverage of $\sim 0.6$. In total there are 40 optically isolated sectors. The active part of the V0+ is made of a $4 \mathrm{~cm}$ thick EJ-204 plastic scintillator. The back surface of the scintillator is optically 
coupled to a grid of clear optical fibers, attached perpendicularly to the scintillator, and spaced by 3-7 mm. The fibers from each cell are bundled together and connected to a Hamamatsu fine-mesh PMT (R5924). The measured time resolution of the V0+ is $120 \mathrm{ps}$ for the internal rings and $300 \mathrm{ps}$ for the external ring. The lower resolution of the external ring is due to its large area and sparser fiber grid density. The V0+ makes a crucial contribution to the FIT functionality, especially in terms of centrality and event plane resolution (see Fig. 2).
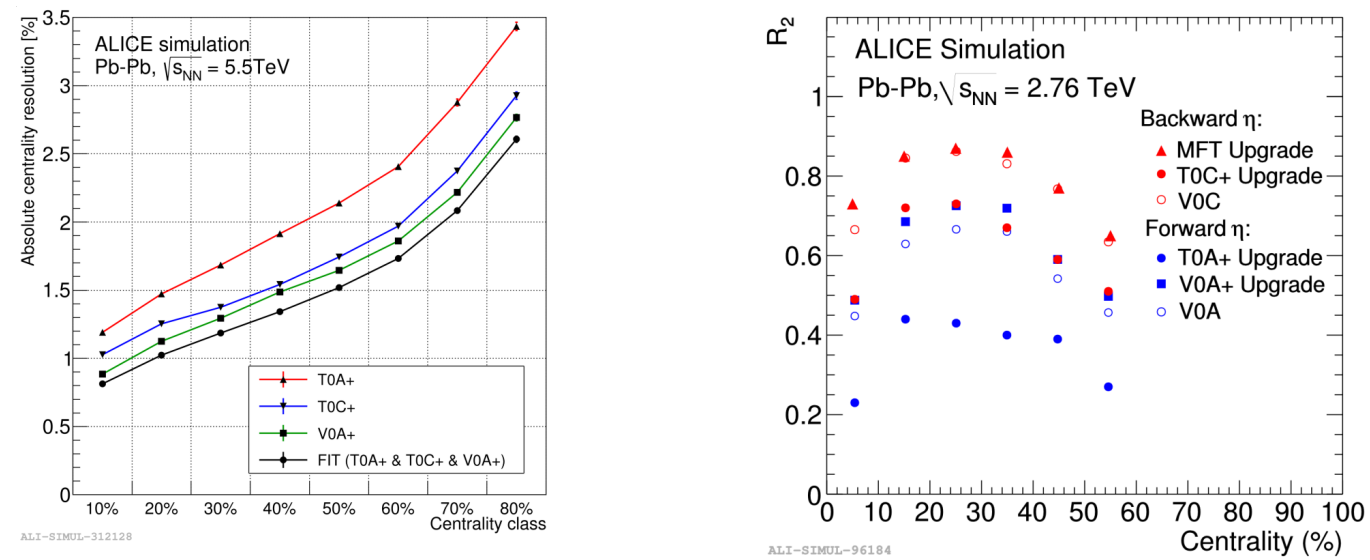

Figure 2: Left: Centrality resolution of different components of FIT as a function of centrality simulated for Run 3 energy. Right: Event plane resolution as a function of centrality, simulated in Run 2 conditions.

The FIT will function as the main ALICE luminometer with a direct feedback to the LHC. It will provide a clean minimum-bias trigger by rejecting beam-gas interactions and ultra peripheral collisions. The overall trigger efficiency for pp collisions will be improved with respect to the present ALICE apparatus. The FIT will be capable of online vertex position determination with a resolution along the beam direction better than $17 \mathrm{~mm}$. It will also generate centrality-based triggers. The FIT data will be used to determine unbiased forward multiplicity needed to extract the centrality and the reaction plane for each event. The FIT will also provide a precise collision time for the Time-of-Flight-based particle identification.

\section{Muon Forward Tracker (MFT)}

A simplified drawing of the MFT [3] is shown in Fig. 1 (middle). The MFT covers the pseudorapidity of $-3.6<\eta<-2.5$. It consists of 5 disks located between the interaction point and the hadron absorber of the Muon Spectrometer. The disks are instrumented on both sides with ALICE pixel detectors (ALPIDE). The ALIPIDE chips are used for the upgrade of both the ITS and the MFT. The single pixel size is $27 \times 29 \mu \mathrm{m}^{2}$. The small thickness of $50 \mu \mathrm{m}$ helps to reduce the material budget down to $0.7 \%$ of a radiation length per disk. The detector is also radiation-hard and will be able to withstand up to $400 \mathrm{krad}$, which is about 10 times the expected dose collected during the LHC Run 3 and Run 4 operation. The MFT readout will be capable of recording $\mathrm{Pb}-\mathrm{Pb}$ collisions at the target interaction rate of $50 \mathrm{kHz}$ and pp collisions - up to $1 \mathrm{MHz}$.

The physics motivation of the MFT detector [3] is the measurement of beauty production at forward rapidity using $\mathrm{J} / \psi$ mesons or single muons from b-hadron decays, as well as the improvement of the measurements of prompt $\mathrm{J} / \psi$ and $\psi(2 \mathrm{~S})$ states. Figure 3 shows the comparison between 

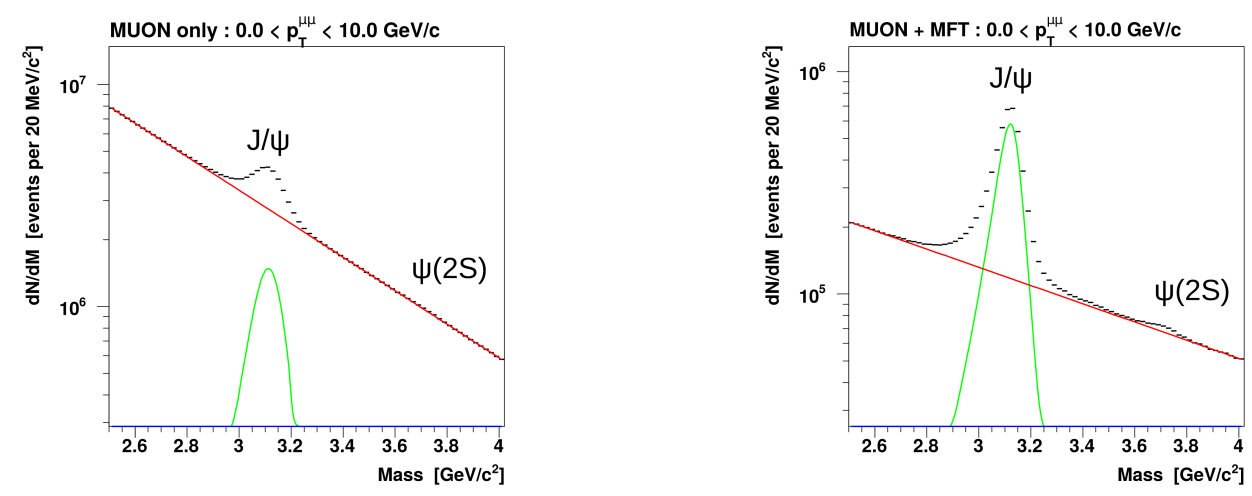

Figure 3: Comparison of the signal-to-background ratio for $\mathrm{J} / \psi$ and $\psi(2 \mathrm{~S})$ without (left) and with the MFT (right). The plots show result of simulations of $10 \%$ most central $\mathrm{Pb}-\mathrm{Pb}$ collisions at $\sqrt{s_{N N}}=5.5 \mathrm{TeV}$ normalised to the expected integrated luminosity of $10 \mathrm{nb}^{-1}$ [4].

simulated performances of present-day Muon Spectrometer only (left) and after the installation of the MFT (right). The improvement of signal-to-background ratio reaches one order of magnitude, which largely increases the statistical significance of the $\psi(2 S)$ signal.

\section{Forward Calorimeter (FoCal)}

The FoCal is a Forward Calorimeter [5] proposed for installation during LS3. Its outstanding feature would be the inclusion of two high-granularity layers, made of Monolithic Active Pixel Sensors (MAPS), which improve tracking resolution and allow for discrimination between direct and decay photons, and provide a two-shower separation. The FoCal would extend the physics scope of the ALICE detector by providing measurements of direct photons down to Bjorken- $x 10^{-6}$ in $\mathrm{p}-\mathrm{Pb}$ collisions. This will yield important information on high-density gluon dynamics in $\mathrm{Pb}$ nuclei at unprecedented low $x$. It will also constrain nuclear parton distribution functions (PDF) and may shed new light on possible non-linear PDF evolution effects and the onset of gluon saturation.

\section{Conclusions}

The MFT and FIT detectors will be installed in ALICE in early 2020. The detectors reach or exceed the design parameters required by the physics objectives. The FoCal has been proposed to be added to ALICE during the Long Shutdown 3 (2024-25). If implemented it promises new and exciting results related to nPDFs and gluon dynamics at very low Bjorken- $x$.

\section{References}

[1] B. Abelev et al. Upgrade of the ALICE Experiment: Letter Of Intent. J. Phys., G41:087001, 2014.

[2] W. H. Trzaska. New Fast Interaction Trigger for ALICE. Nucl. Instrum. Meth., A845:463-466, 2017.

[3] J. Adam et al. Technical Report CERN-LHCC-2015-001. ALICE-TDR-018, CERN, Jan 2015.

[4] Technical Report CERN-LHCC-2013-014. LHCC-I-022-ADD-1, CERN, Geneva, Aug 2013.

[5] N. van der Kolk. JINST, 13(03):C03030, 2018. 